\title{
A Nonlinear Optimization Model of Advertising Budget Allocation across Multiple Digital Media Channels
}

\author{
Sung-Hyuk Park \\ KAIST College of Business \\ sunghyuk.park@kaist.ac.kr \\ Kitae Kim \\ KAIST College of Business \\ kitae.kim@kaist.ac.kr
}

\begin{abstract}
The goal of advertisers in the digital marketing industry is to optimize their advertising budgets. Such a budget allocation problem plays a key role in maximizing advertising performance from different marketing channels under planned advertising investment. This study aimed to design a budgetperformance-based nonlinear programming model to find an optimized solution for the advertising budget allocation problem. The empirical analysis results of $a$ leading e-business company's advertising performance data show that the proposed non-LP model generates an optimized solution. The proposed model allows marketers to simulate expected advertising returns, such as conversions or revenues from different channels within their budget constraints.
\end{abstract}

\section{Introduction}

The global digital advertising market is projected as $\$ 154$ billion (Statista $2020^{1}$ ) and is conducted in a manner that runs ads on a variety of digital media, such as Google, Facebook, and Twitter. These leading digital advertising companies provide fully automated advertising planning, execution, and evaluation processes on their platforms. This allows marketers to try a quantitative approach to maximize the utility base from the resulting data. Specifically, for each media, for a given time period, the total amount of marketing budget, total number of visits, conversions, and revenue amounts are provided through the media company's advertising platform. From an advertiser perspective, it is possible to track every individual user's behavior history data, not as summarized

\author{
Minhyung Lee \\ KAIST College of Business \\ mh1323@kaist.ac.kr \\ Dongwook Shin \\ HKUST Business School \\ dwshin@ust.hk
}

statistics [1] due to the low tracking costs of the digital economy. Despite the advantages of the digital environment, advertisers are still experiencing problems in that there is no single advertising media that provides an integrated view of advertising performance analysis that includes its competitors (e.g., Facebook ads do not evaluate Google ads). Facebook only reports performance reviews of numbers and insights conducted on its own advertising platform and cannot evaluate other marketing channels' performance conducted by the same advertiser. Therefore, advertisers whose budget is usually spread across multiple channels are not able to gain scientific evaluation of the performance of each channel with an integrated view.

For digital marketing, advertisers seek to determine how much advertising budget is best spent on each medium to run ads across many media channels in the market rather than relying solely on a single medium. Focusing on the duplication of users between media channels, a recent study was conducted to measure the degree of contribution that influences the same user for each medium when purchasing products by viewing advertisements from different media [2]. These studies present a contribution model rather than an advertising budget optimization perspective and introduce how to measure performance, so they are limited by their failure to provide a specific answer to the budget optimization sought by the advertisers. To fill the gap between academics and practical markets, this study proposes a nonlinear programming model for solving a budget allocation problem with multiple channels.

When designing the objective function and constraints, in practical terms, it was intended to apply the same essential factors that business practitioners

\footnotetext{
${ }^{1}$ Digital Advertising Report. Retrieved from https://www.statista.com/study/38338/digital-advertisingreport-search-advertising/
} 
use to refer to the budget allocation process. Specifically, the objective function of the model is designed to contain conversions or revenue as a result of a digital marketing campaign, and the minimum budgets for each channel are considered as constraints. For empirical analysis, using real-world data collected by a leading e-business company in Korea that has 12 different digital marketing channels, we analyzed budget-performance curve fitting and non-linear optimization to solve the budget allocation problem. Compared to the result of conventional budget allocation strategy, our optimization result show more efficient budget allocation and this shows that our model successfully determined the optimized portfolio.

\section{Related Literature}

In the field of digital marketing and information systems, the measurement of advertising performance from multiple channels is an important research question. Recently, one study employed the attribution model and diagnosed the quantitative contribution of each advertising channel by analyzing individual users' behavior data [2]. Other scholars have discussed the allocation of advertising budgets from the perspectives of risk and uncertainty [3]. However, there is no welldefined research method that provides guidelines for allocating an optimized budget to different advertising channels. Although it has always been an important research issue, as companies face barriers in distributing advertising budgets in response to the business cycle [4], prior studies have been limited to optimizing the annual budget for the overall marketing period. Hence, attempts to optimize the budget for each digital channel have not been managed well. In terms of a return on advertising spending, each digital channel has a unique pattern of efficiency because channel users and their service usage or content consumption behaviors differ from those observed in other channels. Although the level of efficiency is unique to each channel, the relationship between costs and returns is universal. Thus, it is necessary to design an optimization model for advertising budget allocation, considering the uncertainty of each advertising channel.

Budget allocation models have also been addressed in previous studies. Research on the expected performance against advertising costs has been steady, with the relationship between performance and advertising costs being addressed. Operations research literature takes a mathematical approach to build the advertising response function. A pioneering study suggested a simple mathematical model called the Vidale-Wolfe model [5]. However, empirical evidence for the effectiveness of the proposed model was not provided and the model has a limitation in that it assumes a non-stochastic process. To overcome this limitation, the Sethi model was developed, which is a stochastic extension of the Vidale-Wolfe model [6]. A recent study extended the Vidale-Wolfe model for advertising portfolio optimization across multimarkets with different goals and various constraints [7]. On the other hand, marketing research takes economic theory and suggests that the relationship between advertising costs and performance follows the law of diminishing returns [8]. It is well known that the function of advertising is concave (increasing with diminishing returns) or s-shaped [9-12]. Empirical evidence also supports the idea that the ad response curve is concave [13]. An empirical study on the effectiveness of banner ads on online purchase patterns has shown that the probability of purchasing and the total number of ad exposures follow the law of diminishing returns [14]. In this study, a logarithmic function was used to capture the diminishing returns of purchases. Logarithmic function has also been used by practitioners to model the advertising response function between revenue and ad cost [15]. Therefore, following the marketing literature, we expect advertising performance against spending to follow a $\log$ function. Our budget allocation model captures the law of diminishing returns, for which the budget optimization problem is formulated as a (strictly) convex optimization problem, allowing for a (unique) optimal solution that is tractable both theoretically and numerically [16].

Furthermore, budget allocation and estimation of advertising efficiency have been conducted mostly from longitudinal perspectives [17-20]. This model estimates performance by comparing the budget allocation output efficiency according to the amount of input of decision-making units within a channel using non-parametric linear planning. Thus, it is difficult to check the statistical significance and is not applicable when the objective function is nonlinear. Using a parameter-based nonlinear model, this study proposes a new optimal budget allocation model that considers the performance concurrency among different channels using an empirical dataset including the advertising budget and performance.

\section{Data}

\subsection{Data Description}

We used the firm-level dataset of advertising budget and marketing performance of an e-commerce company for 12 different digital advertising channels, 




Figure 1. Examples of Budget-Performance Curve with Diminishing Returns

including Google and Facebook. These 12 channels were separated into two channel groups: a website advertising group (three channels) and an application marketing group (nine channels). The dataset includes the monthly advertising budget and number of conversions in each channel. The observation period was from January 2019 to January 2020. The average value of the total monthly budget was USD 35,456 and the average monthly conversion approximately 1,626. The average monthly budget and number of purchases are listed in Table 1.

Table 1. Descriptive Statistics

\begin{tabular}{|c|r|r|r|}
\hline Variables & \multicolumn{1}{c|}{ Mean } & \multicolumn{1}{c|}{ Min } & \multicolumn{1}{c|}{ Max } \\
\hline Budget & $\$ 35,456$ & $\$ 598$ & $\$ 297,973$ \\
\hline Conversion & 1,626 & 16 & 5,906 \\
\hline
\end{tabular}

\subsection{Diminishing Return Budget Curve}

As suggested in Section 2, the advertising budget curve follows concave (e.g., log) function with the law of diminishing returns [9, 10] The budget curve, by nature, has a diminishing return distribution. Although the absolute value of conversions increases as the ad budget increases, the rate of increment decreases. To test whether our dataset also follows a diminishing return curve, we drew a scatter plot to check the trend of the conversions by ad budget. As described in Figure 1, conversions of three major channels (channel
$\mathrm{A}, \mathrm{B}$, and $\mathrm{C}$ ) follow a diminishing return curve with $\mathrm{R}^{2}$ of $0.49,0.81$, and 0.90 , respectively, which means that the extent of change in conversions decreases as advertising budgets increase. Accordingly, we assumed that each model follows a log function.

\section{Methodology}

\subsection{Budget Allocation Process}

The process of optimizing budget allocation was separated into four steps. First, we collected both the performance and budget data of each digital advertising channel and matched them together systematically to check the extent to which conversions occurred against the budget. Second, we put the collected dataset into a Python module using the Pandas library and drew scatter plots of our dataset and checked the scatter plots' diminishing return shape using the Matplotlib library. Figure 1 shows an example of a scatter plot. According to shape, we estimated the trend line of advertising performance against budget using the curvefit function of the Python SciPy library. By applying this function, we estimated the coefficients of the advertising performance trend lines of each channel. Third, we set the nonlinear objective function to solve budget optimization problems using the estimated budgetperformance curve in stage 2. Using MOSEK, we 


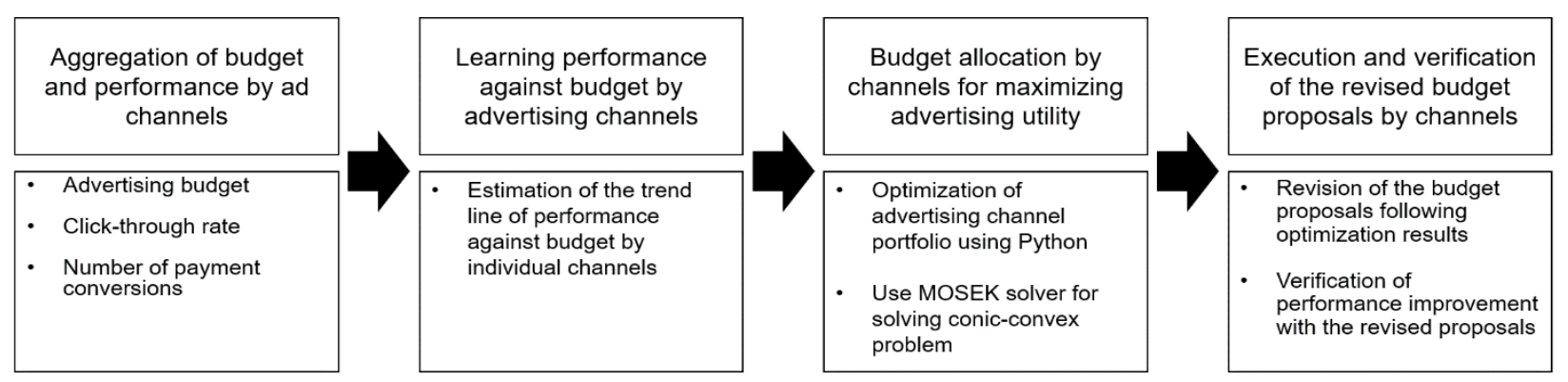

Figure 2. Summary of Analysis Process

found the optimal solution to maximize advertising utility against the budget. The MOSEK solver is appropriate for finding a conservative solution with convex-cone programs [21] and we followed the method suggested by Diamond and Boyd (2016) [22] using the Python CVXPY library.

Finally, in stage 4, we checked which channel budgets could be reduced and which should be increased and planned to apply a revised budget proposal to verify how executing our optimized budget plan changed advertisement performances. The process described above is summarized in Figure 2.

\section{Solver}

The MOSEK solver, which was developed by the Danish Advanced Optimization Software Development Company, MOSEK ApS, is a specialized solver for solving conic optimization problems. The solver has been widely used even compared to IBM's CPLEX, which is the most frequently used commercial solver. Specifically, the MOSEK solver has been applied to optimization for financial portfolios, smart grid systems, and threedimensional telecommunication network station distribution [23-26].

A solver capable of solving conic exponential optimization (CEO) problems was needed, since the objective function of the advertising budget optimization model in this study was a log function. However, few solvers can solve CEO problems; only ECOS, SCS, and MOSEK are able to solve this type of problem [27]. Among them, ECOS and MOSEK are based on the interior point method, while SCS, which is based on the first-order method, is more appropriate for solving large-scale problems that only need to determine approximate solutions rather than accuracy. We tested the three solvers on the optimization model of this study and selected MOSEK as it would most accurately solve the problem.

\subsection{Conversion Maximization Model}

The main goal of this study was to develop nonlinear optimization models and to find an optimal solution for advertising budget allocation by channels using a specific solver to maximize advertising utility. To measure advertising utility and set up an objective function, we selected an advertising performance, which is a general index defining the effect of advertisements. This variable is expressed as follows:

$$
\left.{\text { Advertising } \text { Utility }_{i}=f\left(\text { Performance }_{i}\right)}\right) \text {, }
$$

To measure advertising performance, we selected conversions in each advertising channel as a representative key performance index (KPI). We suggest a nonlinear optimization model as follows. First, according to the law of diminishing returns, the optimal digital advertising budget allocation problem can be formulated as follows:

$$
\begin{aligned}
\text { Purchase }_{i}=\alpha_{i} & +\beta_{i} \ln \left(\text { Budget }_{i}\right) \\
& +\varepsilon_{i}, \quad \text { for all } i,
\end{aligned}
$$

where $i$ indicates the $i$-th digital marketing channel, and $\alpha_{i}$ and $\beta_{i}$ are estimated from the monthly dataset of digital channel $i$.

Second, we set our budget allocation problem for maximizing advertising revenue as follows:

$$
\operatorname{Max} \sum_{i=1}^{N} E\left[\text { Purchase }_{i}\right]
$$

$$
\begin{gathered}
\text { s.t. } \sum_{i=1}^{N} \text { Budget }_{i} \leq \text { MonthlyBudget } \\
\text { Budget }_{i} \leq \text { BudgetCap }_{i}, \text { for all } i \\
E\left[\text { Purchase }_{i}\right] \geq 0, \text { for all } i,
\end{gathered}
$$

where MonthlyBudget indicates the predetermined total budget of a company's annual marketing plan. Here we set MonthlyBudget at USD 416,667 (KRW 500,000,000), because the average value of the total monthly advertising budget is USD 333,333 (KRW 400,000,000), which increases in the peak season (November) to USD 500,000 
(KRW 600,000,000). The value of USD 416,667 is the mean value of these two values.

BudgetCap is the budget limit on each channel. We set the budget cap on each channel to prevent extrapolation. To test the effect of the budget cap, we compare the no-budget cap model and budget capped model. In the no-budget cap model, we set BudgetCap to zero, while BudgetCap is set to the maximum advertising spending on one channel in the budget capped model.

The budget allocation problem is a (strictly) concave maximization problem, for which there exists a unique optimal solution characterized by the Karush-KuhnTucker conditions. Further, the objective function and the constraints are essentially independent of time $t$, so the multi-period problem above can be reduced to a single-period problem; hence, we report the optimal budget allocation for a single period in the following.

Figure 3 summarizes the inputs and outputs of our model. The inputs for the ad response model are historical budget allocation amounts and conversions (i.e., purchases) per budget for each advertising channel. The ad response prediction model and budget constraints are the inputs for the budget portfolio optimization model. Finally, the optimization model outputs the optimal budget for each advertising channel.

\section{Results}

Figures 4 and 5 show the optimization results of the proposed budget allocation model without budget cap. To compare the optimized performance of each channel, we show our results using a pie chart. Figure 4 shows the optimization results of the proposed budget allocation model, and Figure 5 shows the expected conversions based on the budget allocation results summarized in Figure 4. As each channel has a unique diminishing return budget-performance curve, the portion of conversions against the portion of the budget is unique. Our results show that advertising efficiency differs across the channels. Channels 10 and 11 account for 13.3 and 26.5 percent of the overall budget, respectively, but 7.8 and 15.3 percent of the conversion rate, respectively, which is considerably lower. Channels 5 and 8 account for 14.1 and 3.2 percent of the total advertising budget, respectively, but they show higher total conversion rates (19.1 and 9.0 percent, respectively). In other words, the advertising performance in these two channels (i.e., channels 5 and 8) is more efficient than the others (i.e., channels 10 and 11).

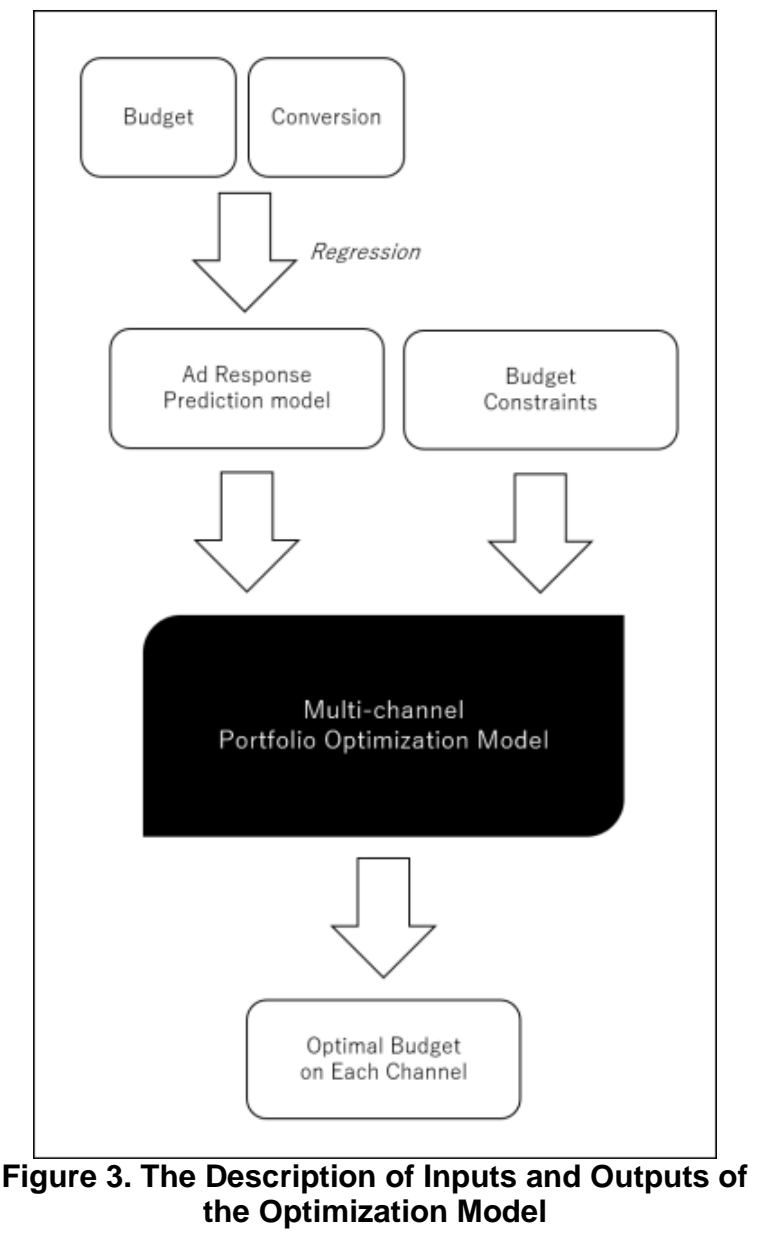

Table 2 reports both the conventional allocation result and the optimization results when there is no budget cap or budget cap is the past maximum budget. When there is no limit to the monthly budget in each channel, most of the budget is allocated to channel 11 as described in column 6 . It occupies the entire budget by $26.54 \%$. However, when we set the budget limit as the past maximum budget in each channel, the allocation of the advertising budget is different from that of the no-budget-cap model. In the budget-cap model, channels 10 and 11 occupy most of the budget, while Channel 3 only occupies $1.20 \%$. The detailed percentage of the budget in each channel is shown in Figure 6.

As the model without a budget cap derives a more progressive result, the advertising performance has improved by $33.97 \%$ compared to the conventional allocation model. The model with a budget cap suggests more conservative results compared to the model without; that is, the advertising performance has improved by $26.85 \%$ (less than that of the model without a budget cap). 
Table 2. Optimization Results

\begin{tabular}{|c|r|r|r|r|r|r|}
\hline & \multicolumn{2}{|c|}{$\begin{array}{c}\text { Conventional } \\
\text { Allocation }\end{array}$} & \multicolumn{2}{c|}{$\begin{array}{c}\text { Optimization Model Capped } \\
\text { by Past Max Budget }\end{array}$} & \multicolumn{2}{c|}{$\begin{array}{c}\text { Optimization Model without } \\
\text { Budget Cap }\end{array}$} \\
\hline & \multicolumn{1}{|c|}{ Budget } & Conversion & \multicolumn{1}{c|}{ Budget } & \multicolumn{1}{c|}{ Conversion } & \multicolumn{1}{c|}{ Budget } & \multicolumn{1}{c|}{ Conversion } \\
\hline Total & $\$ 416,666.66$ & 17,518 & $\$ 416,666.66$ & 22,221 & $\$ 416,666.64$ & 23,468 \\
\hline Channel 1 & $\$ 17,924.05$ & 493 & $\$ 22,501.68$ & 553 & $\$ 15,947.78$ & 462 \\
\hline Channel 2 & $\$ 5,275.84$ & 1,197 & $\$ 8,328.44$ & 1,562 & $\$ 48,004.79$ & 2,960 \\
\hline Channel 3 & $\$ 32,417.79$ & 778 & $\$ 5,018.91$ & 668 & $\$ 3,552.67$ & 647 \\
\hline Channel 4 & $\$ 4,664.94$ & 886 & $\$ 13,914.40$ & 1,342 & $\$ 25,103.02$ & 1,589 \\
\hline Channel 5 & $\$ 11,579.63$ & 2,886 & $\$ 30,553.20$ & 3,836 & $\$ 58,805.75$ & 4,476 \\
\hline Channel 6 & $\$ 6,197.59$ & 734 & $\$ 23,138.60$ & 1,094 & $\$ 16,388.79$ & 1,000 \\
\hline Channel 7 & $\$ 6,713.07$ & 1,124 & $\$ 14,880.99$ & 1,414 & $\$ 21,898.98$ & 1,555 \\
\hline Channel 8 & $\$ 16,336.47$ & 68 & $\$ 18,657.82$ & 2,192 & $\$ 13,209.41$ & 2,116 \\
\hline Channel 9 & $\$ 5,366.45$ & 1,490 & $\$ 10,667.02$ & 1,576 & $\$ 7,547.51$ & 1,533 \\
\hline Channel 10 & $\$ 72,709.17$ & 2,080 & $\$ 77,942.29$ & 2,144 & $\$ 55,221.79$ & 1,827 \\
\hline Channel 11 & $\$ 225,793.75$ & 4,896 & $\$ 156,015.82$ & 4,217 & $\$ 110,589.29$ & 3,584 \\
\hline Channel 12 & $\$ 11,687.91$ & 885 & $\$ 35,047.49$ & 1,623 & $\$ 40,396.85$ & 1,719 \\
\hline \multicolumn{2}{|c|}{$\begin{array}{c}\text { Performance Improvement over } \\
\text { Conventional Allocation Model }\end{array}$} & $\underline{\mathbf{2 6 . 8 5 \%}}$ & & $\underline{\mathbf{3 3 . 9 7 \%}}$ \\
\hline
\end{tabular}

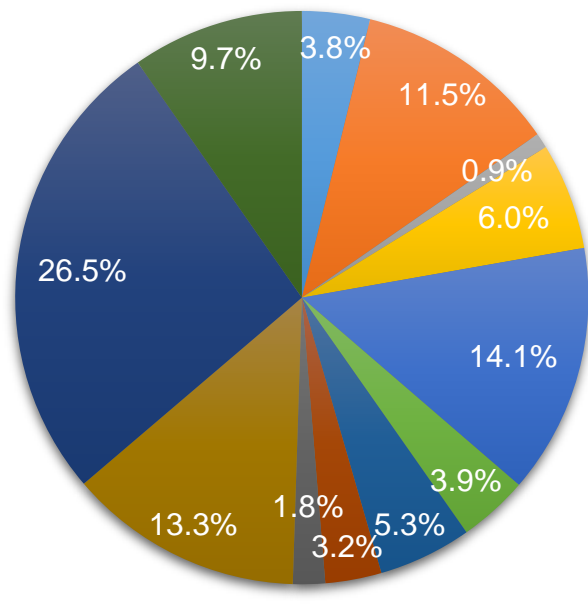

-Channel 1 Channel 2 Channel 3

-Channel 4 Channel 5 Channel 6

-Channel 7 Channel 8 Channel 9

-Channel 10 a Channel 11 Channel 12

Figure 4. Optimized Results of Budget Allocation without Budget Cap

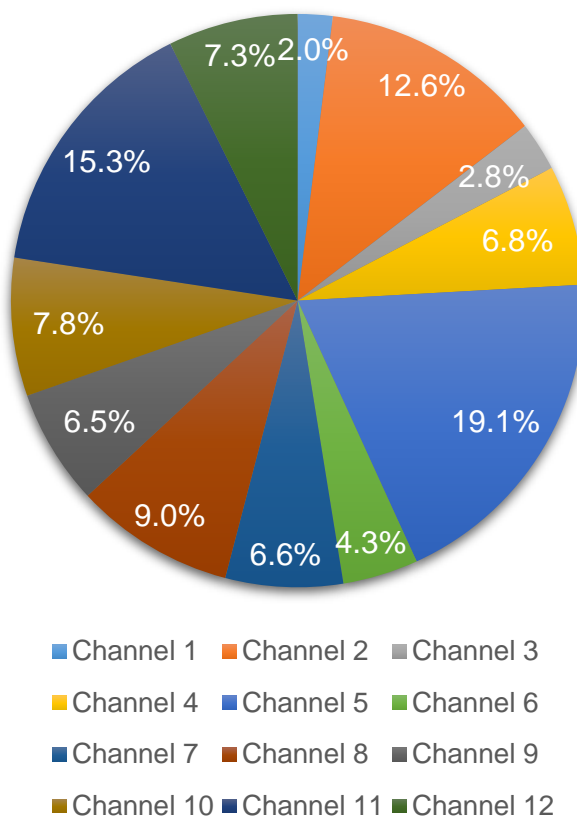

Figure 5. Expected Conversions without Budget Cap 


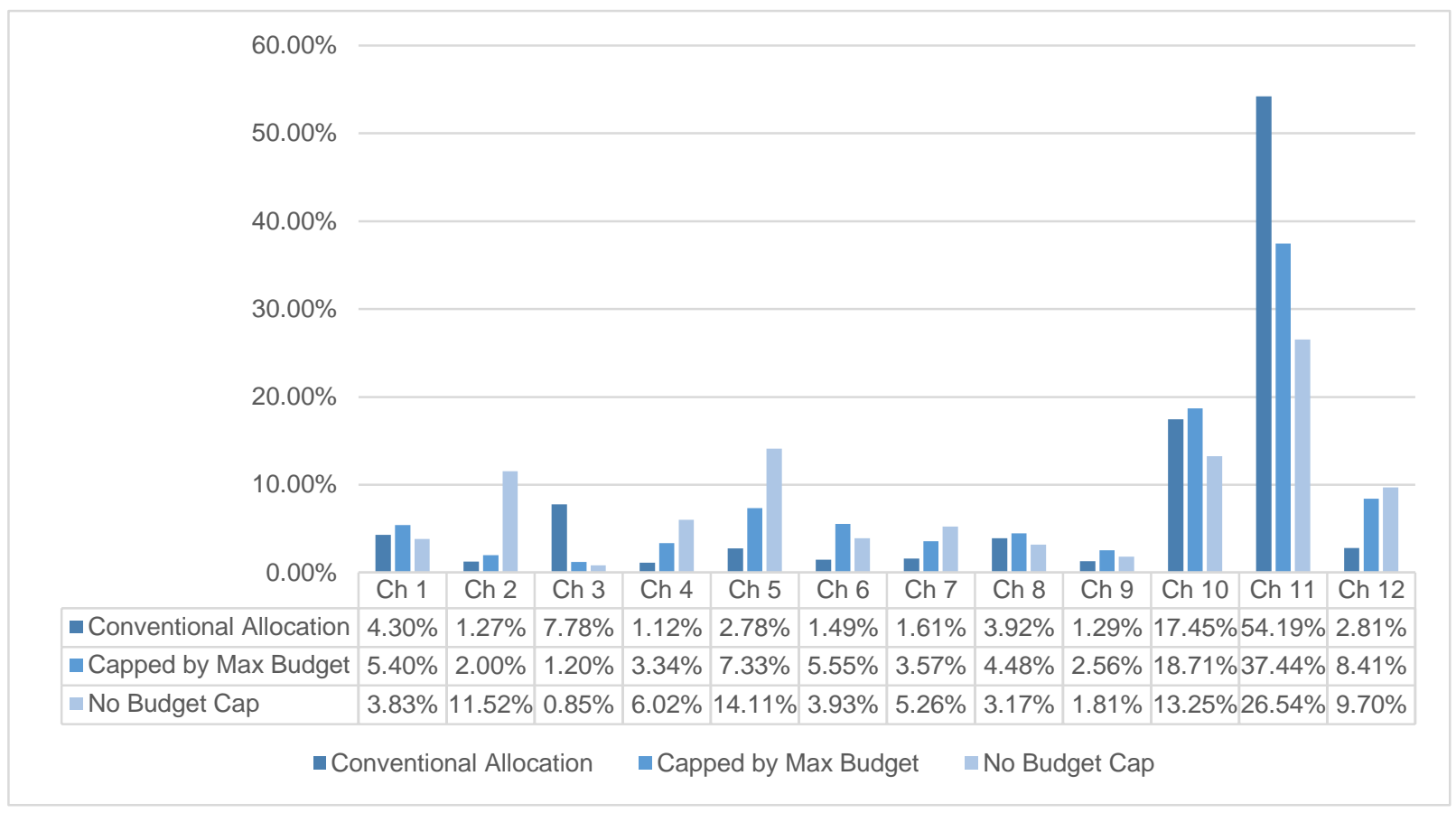

Figure 6. Comparison of Budget Allocation Results based on Budget Cap

\section{Conclusion}

Using a real advertising performance dataset from different channels, as a preliminary measure we first suggested an optimization model for digital advertising budget allocation. This is the first study to explain the methodology for optimizing the budget amount of different digital marketing channels and empirically analyze the optimal marketing budget allocation. We suggested budget allocation optimization models from a representative digital marketing index perspective, that is, advertising performance. Our results indicate that optimal results for performance were not consistent with those for brand awareness. We addressed the necessity of reducing the budget for inefficient digital channels and augmenting that of efficient digital channels, considering both performance and brand awareness. To verify our results, we plan to execute our optimal budget portfolio and investigate how marketing performance changes after executing the newly developed budget allocation strategy.

However, there are some limitations to this study. First, we used a dataset from a single company. The digital advertising strategy does not differ much among companies, but will be more robust if our model can be analyzed with datasets from other advertising companies. Second, we did not consider seasonality in the models. It was difficult to take seasonality into account because the company was not running advertisements to a certain level for a specific period of time on all digital ad channels, but rather on a particular ad channel at a particular time. To check how seasonality affects budget allocation, it would be necessary to employ the optimization model again with a larger-scale dataset from multiple companies over different periods of time. Third, detailed information about the cost per advertising execution was not considered in our model. The advertising billing system varies from channel to channel, and detailed billing information was not provided as companies do not collect this information in the form of databases. Finally, we did not reflect the interdependency among digital advertising channels owing to the lack of information about the relationship between channels. In the future, we will collect billing and cost information from each advertising channel to consider channel-specific effects in our model. We will also collect user-behavior log data from advertising websites and suggest a strategy enabling the automation of digital channel budget allocation step by step. Finally, we will consider the interdependency of advertising channels with a dataset of channels that advertised simultaneously.

\section{References}

[1] Goldfarb, A., and Tucker, C., "Digital Economics", Journal of Economic Literature, 57(1), 2019, pp. 3-43. 
[2] Ghose, A., and Todri, V., "Towards a Digital Attribution Model: Measuring the Impact of Display Advertising on Online Consumer Behavior", MIS Quarterly, 40(4), 2016, pp. 889-910.

[3] Holthausen Jr, D.M., and Assmus, G., "Advertising Budget Allocation under Uncertainty", Management Science, 28(5), 1982, pp. 487-499.

[4] Peers, Y., Van Heerde, H.J., and Dekimpe, M.G., "Marketing Budget Allocation across Countries: The Role of International Business Cycles", Marketing Science, 36(5), 2017, pp. 792-809.

[5] Vidale, M.L., and Wolfe, H.B., "An OperationsResearch Study of Sales Response to Advertising", Operations Research, 5(3), 1957, pp. 370-381.

[6] Sethi, S.P., "Deterministic and Stochastic Optimization of a Dynamic Advertising Model", Optimal Control Applications and Methods, 4(2), 1983, pp. 179-184.

[7] Wang, X., Li, F., and Jia, F., "Optimal Advertising Budget Allocation across Markets with Different Goals and Various Constraints", Complexity, 2020(2020,

[8] Simon, J.L., and Arndt, J., "The Shape of the Advertising Response Function", Journal of Advertising Research, 1980,

[9] Little, J.D., "Aggregate Advertising Models: The State of the Art", Operations Research, 27(4), 1979, pp. 629667.

[10] Lilien, G.L., Kotler, P., and Moorthy, K.S., Marketing Models, Prentice Hall, 1995.

[11] Broussard, G., "How Advertising Frequency Can Work to Build Online Advertising Effectiveness", International Journal of Market Research, 42(4), 2000, pp. 1-13.

[12] Smith, T.M., Gopalakrishna, S., and Chatterjee, R., "A Three-Stage Model of Integrated Marketing Communications at the Marketing-Sales Interface", Journal of Marketing Research, 43(4), 2006, pp. 564579.

[13] Goldstein, D.G., Mcafee, R.P., and Suri, S., "The Effects of Exposure Time on Memory of Display Advertisements", in Book The Effects of Exposure Time on Memory of Display Advertisements, 2011, pp. 49-58.

[14] Manchanda, P., Dubé, J.-P., Goh, K.Y., and Chintagunta, P.K., "The Effect of Banner Advertising on Internet Purchasing", Journal of Marketing Research, 43(1), 2006, pp. 98-108.

[15] Smith, P. "How Much Should We Spend?" - A Diminishing Returns Analysis," PMG, 2018, retrived from https://www.pmg.com/blog/diminishing-returnsanalysis/?cn-reloaded=1, accessed August 23, 2021.

[16] Ambler, T., and Roberts, J.H., "Assessing Marketing Performance: Don't Settle for a Silver Metric", Journal of Marketing Management, 24(7-8), 2008, pp. 733750.

[17] Büschken, J., "Determinants of Brand Advertising Efficiency: Evidence from the German Car Market", Journal of Advertising, 36(3), 2007, pp. 51-73.

[18] Charnes, A., "Measuring Efficiency of Decision Making Units", European Journal of Operational Research, 3, 1979, pp. 339.
[19] Cheong, Y., De Gregorio, F., and Kim, K., "Advertising Spending Efficiency among Top Us Advertisers from 1985 to 2012: Overspending or Smart Managing?", Journal of Advertising, 43(4), 2014, pp. 344-358.

[20] Luo, X., and Donthu, N., "Benchmarking Advertising Efficiency", Journal of Advertising Research, 41(6), 2001, pp. 7-18.

[21] O'donoghue, B., Chu, E., Parikh, N., and Boyd, S., "Conic Optimization Via Operator Splitting and Homogeneous Self-Dual Embedding", Journal of Optimization Theory and Applications, 169(3), 2016, pp. 1042-1068.

[22] Diamond, S., and Boyd, S., "Cvxpy: A PythonEmbedded Modeling Language for Convex Optimization", The Journal of Machine Learning Research, 17(1), 2016, pp. 2909-2913.

[23] Anand, R., Aggarwal, D., and Kumar, V., "A Comparative Analysis of Optimization Solvers", Journal of Statistics and Management Systems, 20(4), 2017, pp. 623-635.

[24] Ban, G.-Y., El Karoui, N., and Lim, A.E., "Machine Learning and Portfolio Optimization", Management Science, 64(3), 2018, pp. 1136-1154.

[25] Dorostkar-Ghamsari, M.R., Fotuhi-Firuzabad, M., Lehtonen, M., and Safdarian, A., "Value of Distribution Network Reconfiguration in Presence of Renewable Energy Resources", IEEE Transactions on Power Systems, 31(3), 2015, pp. 1879-1888.

[26] Bor-Yaliniz, R.I., El-Keyi, A., and Yanikomeroglu, H., "Efficient 3-D Placement of an Aerial Base Station in Next Generation Cellular Networks", in Book Efficient 3-D Placement of an Aerial Base Station in Next Generation Cellular Networks, IEEE, 2016, pp. $1-5$.

[27] Chen, L., He, L., and Zhou, Y.H., "An Exponential Cone Programming Approach for Managing Electric Vehicle Charging", Available at SSRN 3548028, 2021 